\title{
Case studies of the propagation characteristics of auroral TIDS with EISCAT CP2 data using maximum entropy cross-spectral analysis
}

\author{
S. Y. Ma ${ }^{1 *}$, K. Schlegel ${ }^{1}$, J. S. $\mathrm{Xu}^{2}$ \\ 1* Max-Planck-Institut für Aeronomie, Germany. On leave from Department of Space Physics, Wuhan University, China \\ ${ }^{1}$ Max-Plank-Institut für Aeronomie, D-37189 Katlenburg-Lindau, Germany \\ 2 Dept. of Space Physics, Wuhan University, Wuhan 430072, China
}

Received: 15 January 1997 / Revised: 28 August 1997 / Accepted: 2 September 1997

\begin{abstract}
In this paper case studies of propagation characteristics of two TIDs are presented which are induced by atmospheric gravity waves in the auroral F-region on a magnetic quiet day. By means of maximum entropy cross-spectral analysis of EISCAT CP2 data, apparent full wave-number vectors of the TIDs are obtained as a function of height. The analysis results show that the two events considered can be classified as moderately large-scale TID and mediumscale TID, respectively. One exhibits a dominant period of about $72 \mathrm{~min}$, a mean horizontal phase speed of about $180 \mathrm{~m} / \mathrm{s}$ (corresponding to a horizontal wavelength of about $780 \mathrm{~km}$ ) directed south-eastwards and a vertical phase speed of $55 \mathrm{~m} / \mathrm{s}$ for a height of about $300 \mathrm{~km}$. The other example shows a dominant period of $44 \mathrm{~min}$, a mean horizontal phase velocity of about $160 \mathrm{~m} / \mathrm{s}$ (corresponding to a horizontal wavelength of about $420 \mathrm{~km}$ ) directed southwestwards, and a vertical phase velocity of about $50 \mathrm{~m} / \mathrm{s}$ at $250 \mathrm{~km}$ altitude.
\end{abstract}

Key words Ionosphere $\cdot$ auroral ionosphere · Ionosphere-atmosphere interactions $\cdot$ wave propagation)

\section{Introduction}

Considerable progress has been gained in recent years in the investigation of atmospheric gravity waves (AGWs) and their ionospheric manifestations, the travelling ionospheric disturbances (TIDs), (e.g., recent review by Hocke and Schlegel, 1996). Particularly the incoherent scatter technique provides a versatile tool for studying not only the wave frequencies and vertical wave

Correspondence to: K. Schlegel numbers (e.g., Hearn and Yeh, 1977; Bertin et al., 1983; Schlegel, 1986; Sheen and Liu, 1988; Rice et al., 1988; Natorf et al., 1992; Ma and Schlegel, 1993; Lanchester et al., 1993; Wuttke, 1994), but also details of the fractional variations of various ionospheric plasma parameters such as the electron density, the electron temperature, the ion drift and the ion temperature which constitute the TID (Ma and Schlegel, 1992; Hocke, 1994; Hocke et al., 1996). The inter-relations between the measured TIDs and the AGWs causing them have been explored in detail with suitable models (e.g., the GIFTS Model, Kirchengast 1996a, b; Kirchengast et al., 1992, 1995, 1996).

The propagation of TIDs over large distances cannot be studied with a single instrument, but need experimental facilities which are separated by a suitable distance. This technique has been successfully applied using EISCAT and additional facilities at mid and low latitudes (Morgan, 1983; Crowley and McCrea, 1988; Rice et al., 1988; Williams et al., 1988, 1993). An important quantity for the propagation of TIDs/AGWs is the complete wave vector of the wave-like fluctuations. Whereas its vertical component (or field-aligned component) can be easily derived from data of a radar with a fixed antenna beam, it is more difficult to obtain reliable estimates of the horizontal wave number. The EISCAT CP2 mode was specifically designed to pursue such studies by cycling through four antenna positions (Folkestad et al., 1983). The Japanese middle and upper atmosphere (MU) radar is also capable of observing the ionospheric F-region simultaneously in multiple beams to study horizontal propagation characteristics of TIDs (Oliver et al., 1988). In practice the relatively short distance between these positions in the F-region (of the order of $100 \mathrm{~km}$ ) requires a very accurate determination of the phase front of the TID. Since this front is usually calculated from cross-spectral techniques (e.g., Mauelshagen, 1990; Oliver et al., 1995), a high resolution requires in turn sufficiently long time series. Since TIDs can be traced in the data in general only over two or three wave periods, a proper algorithm for the spectral 
estimates has to be selected. Tests have shown that the maximum entropy method (MEM, Ulrych and Jensen 1974) yields more reliable spectral estimates than the usual FFT algorithm for time series of a length of 2-3 times the wave frequency (Hocke, 1994). We therefore adopted this technique in our study. Within the horizontal wavelength range between about 500 and $1000 \mathrm{~km}$ regarded in our examples the accuracy of the phase front determination proved to be sufficient to obtain reliable estimates of the horizontal wave number. By combining these horizontal components with simultaneously derived vertical wave-number components, the complete vector of the wave propagation can be obtained. A different approach to estimate wave numbers has been taken by Lanchaster et al., (1993): they inserted EISCAT-derived values of wave frequency, field-aligned wavelength and mean neutral wind into Hines' (1960) AGW dispersion relation in order to derive horizontal and vertical wave numbers.

\section{Data and analysis method}

\subsection{EISCAT CP2 data}

In the CP2 mode the EISCAT radar is capable of making measurements during a rapid antenna scan. The scan consists of four positions and is completed in $6 \mathrm{~min}$. The first three positions form a triangle with vertical, south and southeast directions; while the fourth is magnetic field aligned. Thus, the EISCAT CP2 mode can provide some information on the anisotropy of the ionospheric plasma parameter and the horizontal propagation characteristics for wave-like phenomena with certain temporal and spatial scales.

Figure 1 illustrates the geometry of the EISCAT CP2 probing. The azimuth (angle with respect to north, taking clockwise as positive) and elevation of the antenna beam for the four probing positions are:

$$
\begin{array}{llll}
\alpha_{\mathrm{A}}=180.0^{\circ} & \alpha_{\mathrm{B}}=166.5^{\circ} & \alpha_{\mathrm{C}}=133.3^{\circ} & \alpha_{\mathrm{D}}=182.5^{\circ} \\
\beta_{\mathrm{A}}=89.9^{\circ} & \beta_{\mathrm{B}}=62.9^{\circ} & \beta_{\mathrm{C}}=60.4^{\circ} & \beta_{\mathrm{D}}=77.5
\end{array}
$$

With simple geometric calculations, distances between any two probing positions and the azimuth of a line connecting the two positions for each height gate can be obtained. At a height of $300 \mathrm{~km}$, the distances between the positions vary between 65 and $170 \mathrm{~km}$. For medium scale TIDs these distances correspond to a few tenths of the horizontal wavelength.

\subsection{Maximum entropy cross-spectral analysis method}

By means of Fourier cross-spectral analysis the phase difference of a TID at different heights can be calculated. From the height profile of the relative phase, the vertical or field-aligned apparent wave-number of the TID can then be derived, as shown by Ma and Schlegel (1992, 1993), and Hocke et al. (1996). In principle, the horizontal wave-number can also be estimated by Fourier cross-spectral analysis. However, the horizontal wave-number of an AGW or TID is usually much smaller than the vertical one. This results in small phase differences of the wave-like fluctuations of ionospheric parameters at different $\mathrm{CP} 2$ positions and requires high resolution cross-spectral analysis. With the help of the complex maximum entropy spectral analysis (Ulrych and Jensen 1974) it proved possible to obtain phase differences of two time series with sufficient resolution for a data series limited to about 30 data points, corresponding to about $3 \mathrm{~h}$ in the CP2 mode. In our analysis we actually used 40 points $(4 \mathrm{~h})$. So far only Shibata (1987) has applied multi-channel maximum entropy spectral analysis to $\mathrm{HF}$ Doppler data of medium-scale TIDs.

Suppose that $x\left(t_{k}\right)$ and $y\left(t_{k}\right)$ are two real stationary random series, their cross-spectral density (CSD) is a complex random series which can be expressed as

$$
P_{x y}\left(f_{i}\right)=C_{x y}\left(f_{i}\right)+j Q_{x y}\left(f_{i}\right)
$$

with

$C_{x y}\left(f_{i}\right)=\left(P_{b b}\left(f_{i}\right)-P_{x x}\left(f_{i}\right)-P_{y y}\left(f_{i}\right)\right) / 2$
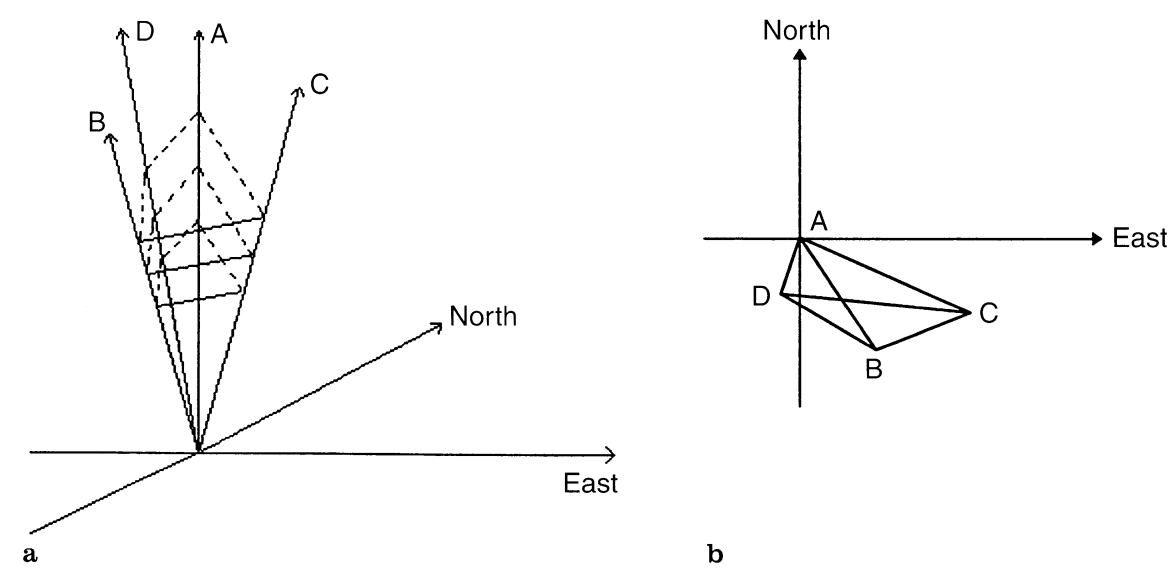

Fig. 1a, b. Illustration of the EISCAT CP2 mode: a the four probing directions of the antenna beam during CP2; b geometric configuration of the four probing positions in a plane at a fixed altitude 
$Q_{x y}\left(f_{i}\right)=\left(P_{a a}\left(f_{i}\right)-P_{x x}\left(f_{i}\right)-P_{y y}\left(f_{i}\right)\right) / 2$

Where $P_{x x}\left(f_{i}\right)$ and $P_{y y}\left(f_{i}\right)$ are auto-power spectral densities of $x\left(t_{k}\right)$ and $y\left(t_{k}\right)$, respectively; $P_{b b}\left(f_{i}\right)$ is the auto-power spectral density of $b\left(t_{k}\right)=x\left(t_{k}\right)+y\left(t_{k}\right)$; $P_{a a}\left(f_{i}\right)$ is the auto-power spectral density of $a\left(t_{k}\right)=x\left(t_{k}\right)+j y\left(t_{k}\right) . \quad P_{a a}, \quad P_{b b}, \quad P_{x x}$ and $P_{y y} \quad$ can be estimated by single channel maximum entropy spectral analysis. The arguments of the CSD yield the spectrum of phase differences between the two real random series and can be calculated from the equation

$$
\begin{aligned}
\delta \Phi(f) & =\arctan \left(\operatorname{Im}\left(P_{x y}(f)\right) / \operatorname{Re}\left(P_{x y}(f)\right)\right) \\
& =\arctan \left(Q_{x y}(f) / C_{x y}(f)\right)+2 n \pi
\end{aligned}
$$

In writing the computer programs for the maximum entropy cross-spectral analysis, contributions by Strand (1977) and Chen (1982) have been used.

Figure 2 a shows as an example the cross-spectral analysis results for a simulated Gaussian random series superimposed on a sinusoidal wave with a wave frequency of $72 \mathrm{~min}$. The signal-to-noise ratio (SNR) was about 9 in this case. Figure $2 b$ shows a similar simulation but here a second sinusoidal wave with a wave frequency of $42 \mathrm{~min}$ has been added. The SNR was about 5 in this case and the phases of the two waves were $+90^{\circ}$ and $-90^{\circ}$, respectively. Based on the central limit theorem the Gaussian series are created from computer-produced independent random numbers following the same uniform distribution. The number of data points of both series was 40 , corresponding to a 4 -h period of CP2 data. It can be realized from Fig. 2 that the CSD estimated by the MEM reproduced well the peaks at 72 and $42 \mathrm{~min}$ and also the phases of both waves. This result which simulates roughly our two TID events confirms the reliability of our algorithm.

\subsection{Estimation of the apparent wave-number vector of the TID}

We assume that the TID has the form of a monochromatic planar wave as:

$$
\begin{aligned}
A(t, \mathrm{r}) & =A_{o} \cos \Phi(t, \mathbf{r}) \\
& =A_{o} \cos (\omega t-\mathbf{k} \cdot \mathbf{r})
\end{aligned}
$$

At two fixed observing points $p 1$ and $p 2$, the sampling starts at the times $t_{1 o}$ and $t_{2 o}$, respectively while sampling at a rate of $1 / \delta t$. The recorded discrete time series of the wave-field at the two positions are

$$
\begin{aligned}
& A_{1}\left(\left(t_{1 o}+n \delta t\right), \mathbf{r}_{1}\right) \\
& \text { and } A_{2}\left(\left(t_{2 o}+n \delta t\right), \mathbf{r}_{2}\right) \quad n=0,1,2, \ldots, m
\end{aligned}
$$

Their phase difference is

$$
\begin{aligned}
\delta \Phi_{12} & =\omega\left(t_{1 o}-t_{2 o}\right)-\mathbf{k} \cdot\left(\mathbf{r}_{1}-\mathbf{r}_{2}\right) \\
& =\omega \delta t_{o}+k_{21} \delta r
\end{aligned}
$$

where $\delta t_{o}$ is the time difference of the sampling start in $p 1$ and $p 2, k_{21}$ is projection of the wave vector $\mathbf{k}$ on the direction from $p 2$ to $p 1$, and $\delta r$ is the distance between $p 1$ and $p 2$. From Eq. (6) we obtain

$$
k_{21}=\left(\delta \Phi_{12}-\omega \delta t_{o}\right) / \delta r
$$
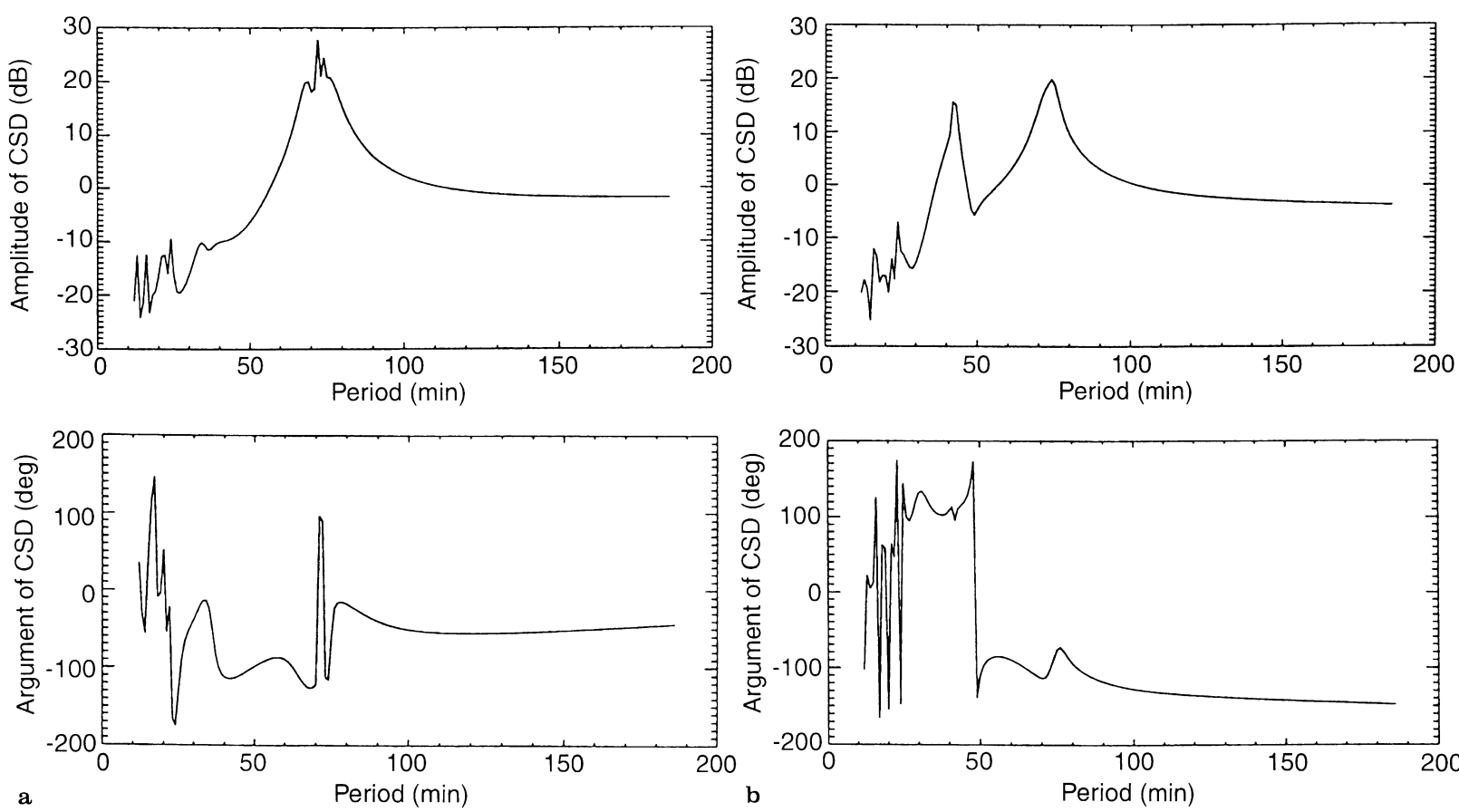

Fig. 2. a Amplitude (top) and argument (bottom) of the cross-spectral density estimated with the maximum entropy method for two time series, composed of a sinusoidal wave with a period of $72 \mathrm{~min}$ and a Gaussian random series. b The same as a but composed of two

sinusoidal waves with periods of $42 \mathrm{~min}$ and $72 \mathrm{~min}$ superimposed on a Gaussian random series. The phases of the two sinusoidal wave pairs are $90^{\circ}$ and $-90^{\circ}$, respectively 
For CP2 data, $\delta r$ and $\delta t_{o}$ of any two probing positions are known. The angular frequency $\omega$ and the phase difference $\delta \Phi$ as a function of $\omega$ can be estimated by maximum entropy cross-spectral analysis, see Eq. (3). Thus, if $p 1$ and $p 2$ are placed in a horizontal plane, a component of the horizontal wave vector along the direction from $p 1$ to $p 2$ can be obtained. Applied to two (non-parallel) directions in the same plane, a value of the horizontal wave vector $\mathbf{k}_{h}$ can be determined, representing an estimate at the center of the probed region. In practice, we omitted those position pairs for which the estimated phase differences turned out to be too small. This situation indicates that the corresponding position pairs happened to fall in nearly the same phase front. If $p 1$ and $p 2$ are aligned along the vertical, the vertical component of the wave-number vector, $k_{z}$, can then be obtained. From the vertical and the horizontal wave-numbers, $k_{z}$ and $\boldsymbol{k}_{h}$, the complete vector of the TID, $\mathbf{k}$, can be reconstructed.

\section{Case analyses: results and discussion}

For these analyses we selected EISCAT CP2 data observed on the magnetically quiet day 14 November, 1990. The Ap index was 1 and $K_{p}$ not larger than 1 . During the time interval of the observed TIDs the ion velocity in the $\mathrm{F}$ region perpendicular to the geomagnetic field was smaller than $50 \mathrm{~m} / \mathrm{s}$, implying a very week convection. Therefore we neglected the effects of the convection electric field.

Figure 3 displays contours of the height dependent power spectral density (PSD) of the $\mathrm{N}_{\mathrm{e}}$-TID during 0730-1130 UT for position A, i.e. the vertical direction. It shows that maximum values of the estimated PSD are distributed over a period range of 60-90 min and an altitude range of $150-350 \mathrm{~km}$. Above about $300 \mathrm{~km}$, the PSD over the period range in question fades out. Figure 4 shows contours of fractional fluctuations of the electron density within a period band of 60-90 min versus time and height. The titled phase front is clearly visible. The phase propagates downward and is thus a typical feature of the TID. Above about $300 \mathrm{~km}$ altitude the TID is fading away.

Figure 5 shows the height profiles of the apparent horizontal wave-number of the $\mathrm{N}_{\mathrm{e}}$-TID (top) and the $\mathrm{T}_{\mathrm{e}}$-TID (bottom) in magnitude (left) and azimuth (right). The profiles plotted as dashed lines are derived from 5 pairs of directions namely $(\mathrm{AB}, \mathrm{BC}),(\mathrm{AB}, \mathrm{AC})$, $(\mathrm{BC}, \mathrm{BD}),(\mathrm{BD}, \mathrm{CD})$, and $(\mathrm{AC}, \mathrm{BC})$. The shortest (AD) from the 6 possible directions $\mathrm{AB}, \mathrm{AC}, \mathrm{AD}, \mathrm{BC}, \mathrm{BD}$, DC was not used (see Fig. 1b). The close agreement of all profiles suggests that the change of the apparent horizontal wave-number over the area surrounded by the four probing points is only small. It also proves the reliability of our analysis method. The profiles plotted by thick solid lines are the averaged $\mathbf{k}_{h}$. It should also be noted that the gross features of the TID in $\mathrm{N}_{\mathrm{e}}$ and in $\mathrm{T}_{\mathrm{e}}$ below $350 \mathrm{~km}$ are quite similar, as should be expected. Above $350 \mathrm{~km}$ the TID is not very well developed as already mentioned. Figure 6 shows the height profile of

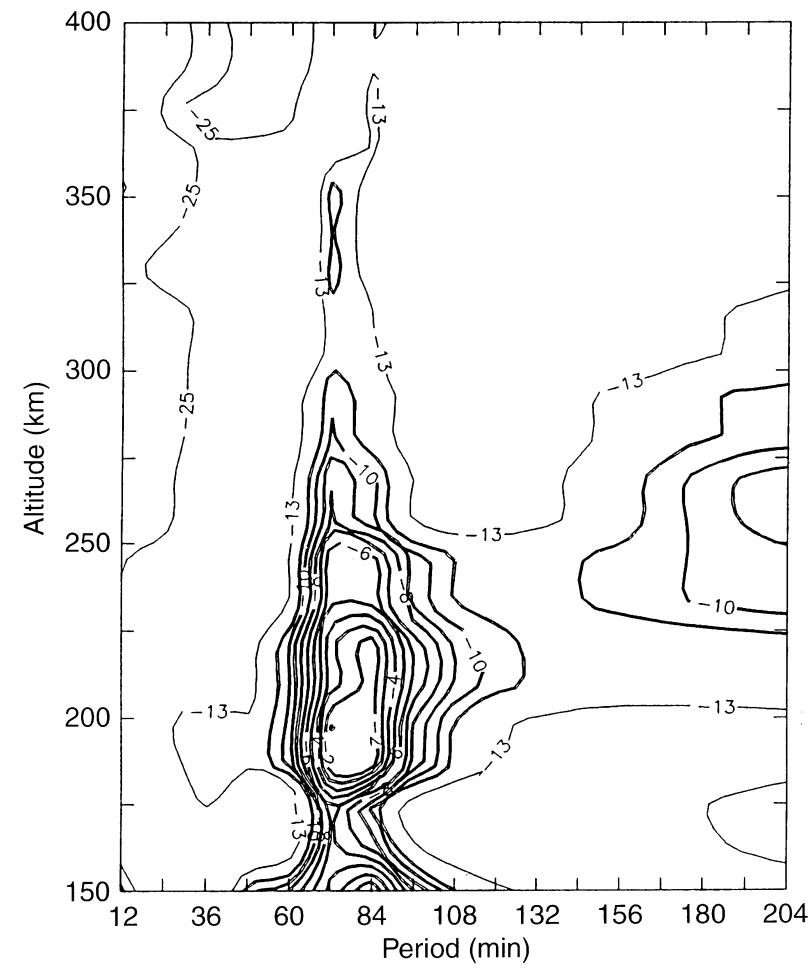

Fig. 3. Contours of the power spectral density of the $\mathrm{N}_{\mathrm{e}}$-TID observed with EISCAT (CP2, position A) on 14 November 1990, from 0730 -1130 UT

the complete apparent wave vector of the $\mathrm{N}_{\mathrm{e}}$-TID in terms of magnitude, azimuth and elevation of $\mathbf{k}$.

We can see from Figs. 5 and 6 that the magnitude of the horizontal wave-number varies slightly with height between $200 \mathrm{~km}$ and $350 \mathrm{~km}$. It shows a mean value of $0.008 \mathrm{~km}^{-1}$ in this height range, corresponding to a horizontal wavelength of about $780 \mathrm{~km}$ and a horizontal phase speed of about $180 \mathrm{~m} / \mathrm{s}$. At $300 \mathrm{~km}$ altitude the magnitude of the apparent vertical wave-number is about $0.027 \mathrm{~km}^{-1}$ and the corresponding vertical wave-

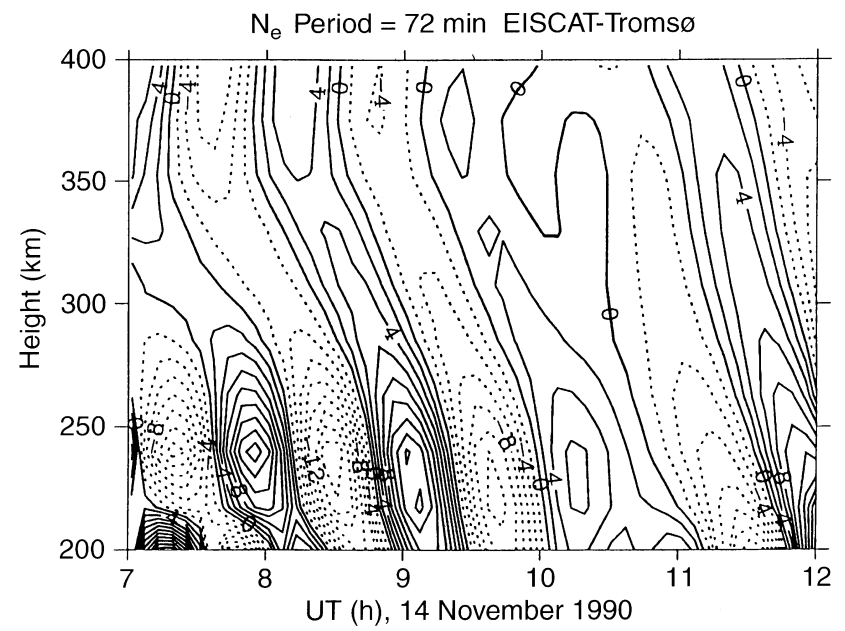

Fig. 4. Electron density fluctuations within a period band of 60-90 min as a function of time and height for the same TID event as shown in Fig. 3 (position A) 

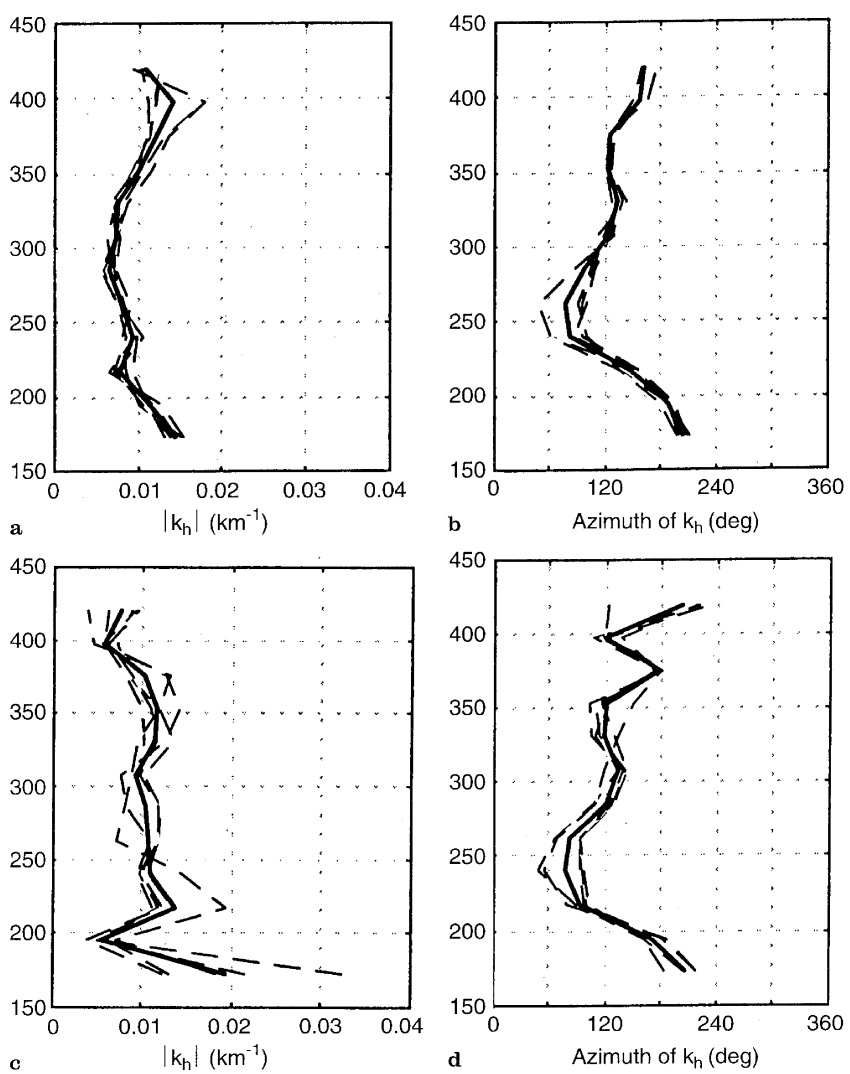

Fig. 5. Magnitude (left column) and azimuth (right column) of the apparent horizontal wave number, $\mathbf{k}_{h}$, versus altitude of the $\mathrm{N}_{\mathrm{e}}$-TID (top row) and the $\mathrm{T}_{\mathrm{e}}$-TID (bottom row) with a period of $72 \mathrm{~min}$. The dashed lines denote the $\mathbf{k}_{h}$ profiles derived from 5 combinations of the 4 different probing positions. The thick solid lines represent the averaged $\mathbf{k}_{h}$

length and phase speed about $230 \mathrm{~km}$ and $55 \mathrm{~m} / \mathrm{s}$, respectively. The apparent vertical wave-number is negative, which means a downward propagation of the phase. These propagation characteristics suggest a moderately large-scale TID. The ratio of vertical to horizontal wave-number magnitude decreases from about 4.3 at $200 \mathrm{~km}$ height to about 2.0 at $350 \mathrm{~km}$, indicating that the propagation of the TID tends to become more horizontal with increasing height. The azimuth changes strongly in the altitude range below about $250 \mathrm{~km}$ from south to east, and stays approximately constant at eastsoutheast above $300 \mathrm{~km}$ altitude (see Fig. 6b). This suggests that the source which excited the AGW is located northwest of the observer, probably in the auroral oval region before dawn.

The other TID studied occurred in the afternoon on the same day. It exhibits a dominant period of about $44 \mathrm{~min}$. Figure 7 shows contours of the fractional fluctuations of the electron density between 12 and 16 UT as a function of altitude. Again, above about $300 \mathrm{~km}$ the TID disappears. The height profiles of the magnitude (left) and the azimuth (right) of the apparent horizontal wave vector are plotted in Fig. 8 (top panels). The profiles are derived using position pairs from the directions $\mathrm{A}, \mathrm{B}$, and $\mathrm{C}$. The horizontal wave-number varies between $0.01 \mathrm{~km}^{-1}$ and $0.02 \mathrm{~km}^{-1}$ corresponding to a mean horizontal wavelength of $420 \mathrm{~km}$ and a horizontal phase speed of about $160 \mathrm{~m} / \mathrm{s}$. The vertical wave-number at $250 \mathrm{~km}$ altitude is about $0.05 \mathrm{~km}^{-1}$, corresponding to a vertical wavelength of about $125 \mathrm{~km}$ and a vertical phase speed of about $50 \mathrm{~m} / \mathrm{s}$. These data characterize a typical medium-scale TID. It can also be seen from Fig. 8 that this afternoon TID propagates southwestwards (azimuth about $230^{\circ}$ ), suggesting a source located probably in the auroral oval after dusk. The lower two panels of Fig. 8 show the height profiles of magnitude and elevation of the apparent full wave vector of this TID. In comparison with the earlier 72-min TID the elevation angle is practically constant with altitude.

The estimation of the apparent wave vector of the TID contains some uncertainties. One cause of uncertainty is the assumption of a planar wave on which Eq. (7) is based. In fact, this assumption is valid for the field far from its source. For TIDs observed in the auroral ionosphere during daytime on a magnetic quiet day the excitation source is usually located far away in an auroral sector before dawn or after dusk. This is the case in our examples and therefore this assumption is valid. The other uncertainty stems from the fact that the horizontal wave vector estimated from CP2 data by
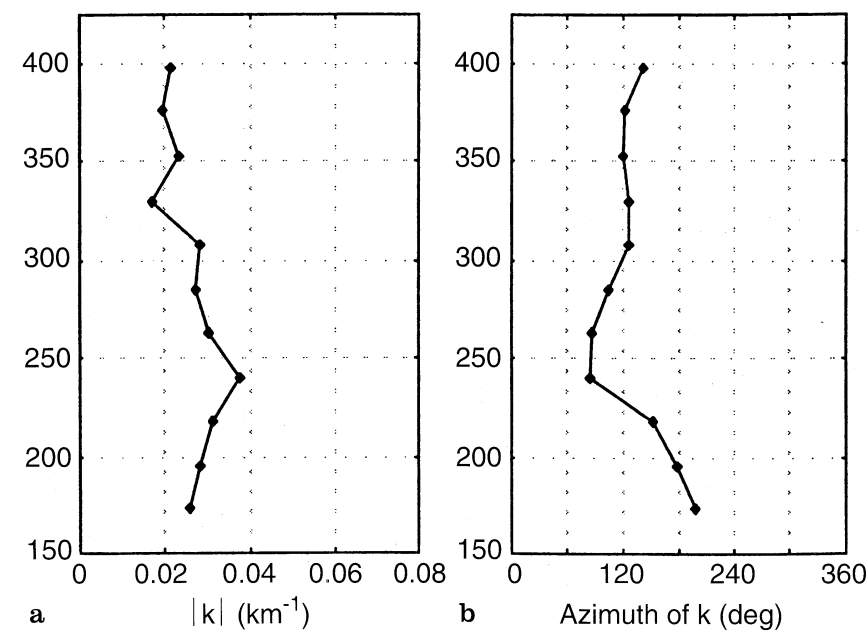

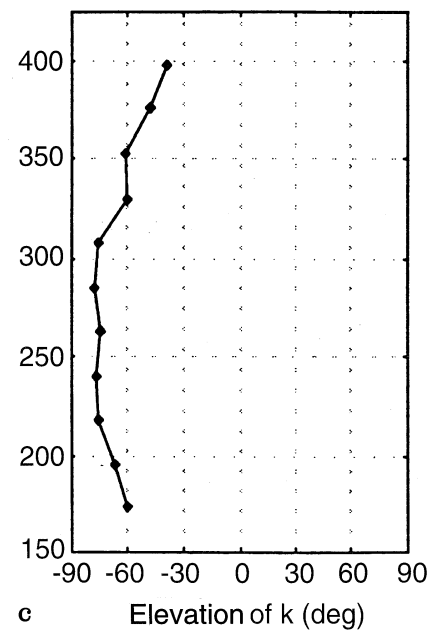

Fig. 6a-c. Height profile of the apparent wave vector, $\mathbf{k}$, of the $\mathrm{N}_{\mathrm{e}}$-TID for a period of about 72 min. a Magnitude of $\mathbf{k} ; \mathbf{b}$ azimuth of $\mathbf{k}$ with north and east direction as positive; $\mathbf{c}$ elevation angle of $\mathbf{k}$ with upward direction as positive 


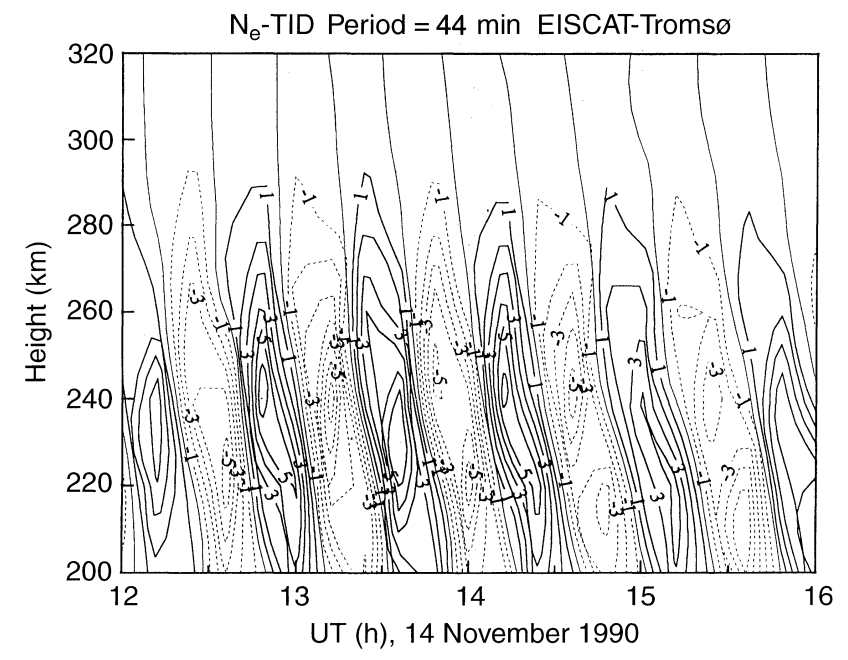

Fig. 7. Contours of electron density fractional fluctuations within a period band of 40-50 min versus time and height for 14 November 1990 (position A)

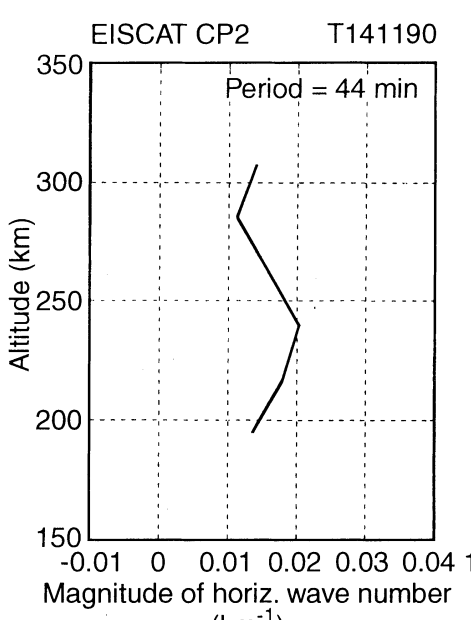
$\left(\mathrm{km}^{-1}\right)$

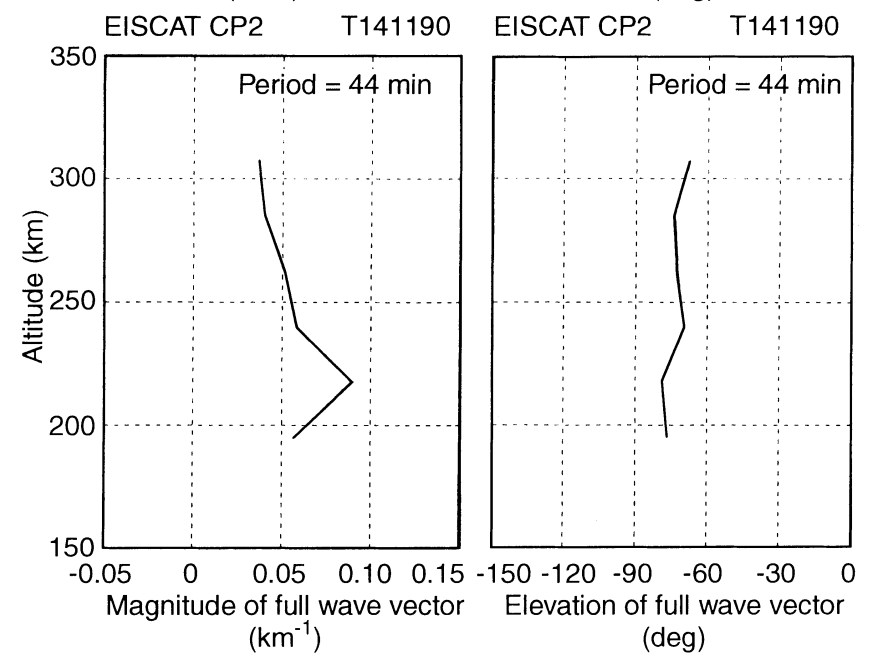

Fig. 8. Height dependent apparent full wave vector of the $\mathrm{N}_{\mathrm{e}}$-TID with a wave period of $44 \mathrm{~min}$. Upper left panel: magnitude of the horizontal wave vector; upper right panel: azimuth of the full wave vector; lower left panel: magnitude of the full wave vector; lower right panel: elevation angle of the full wave vector cross-spectral analysis is valid not exactly for a common probing point, but approximated by a common region around its center. This approximation would also cause errors in the estimation of the horizontal wave vector. In addition, our analysis method of EISCAT CP2 data is valid only for TIDs with a horizontal wavelength below about $1000 \mathrm{~km}$. For example, the distance between position $\mathrm{A}$ and position $\mathrm{C}$ is about $162 \mathrm{~km}$ at a height of $285 \mathrm{~km}$. A horizontal wave vector with $k_{h}=0.0074 \mathrm{~km}^{-1}$ (actual value of the first TID at $285 \mathrm{~km}$ ) and an azimuth of $120^{\circ}$ would have a component with a magnitude of $0.0072 \mathrm{~km}^{-1}$ (corresponding to an apparent wavelength of about $872 \mathrm{~km}$ ) in the direction from position A to position C. For a wave period of $72 \mathrm{~min}$, this would mean a phase difference of $\delta \Phi_{\mathrm{CA}} \sim 33.4^{\circ}$ (corresponding to a time difference about $6.7 \mathrm{~min}$ ) for the phase fronts observed at position $\mathrm{C}$ and position A. Such a phase difference (or time difference) can be easily resolved by our analysis method. In case of a large-scale TID with a horizontal wavelength of $2500 \mathrm{~km}$ (3-6 times the wavelength of our examples), the corresponding time (or phase) difference would become $0.7 \mathrm{~min}$ (or $3.3^{\circ}$ ). Such a small value is very difficult to resolve by the present probing mode and analyzing method. That means, the propagation of large-scale TIDs over large distances cannot be studied with a single instrument, but need experimental facilities which are separated by a suitable distance as mentioned above.

\section{Summary}

EISCAT CP2 data observed on a magnetic quiet day are analyzed to investigate propagation characteristics of a TID. By means of a maximum entropy cross-spectral method, both vertical and horizontal apparent wavenumber of the TID in the auroral $F$ region are obtained as a function of height. The main results can be summarized as follows.

1. For the first time the complete wave propagation vector of TIDs as function of height was derived by using the maximum entropy method in a cross-spectral analysis of EISCAT CP2 data.

2. The resultant apparent wave-number vectors show that their vertical components decrease smoothly with altitude, while their horizontal components are more constant. In our first case the azimuth changes strongly at heights below $250 \mathrm{~km}$. The mechanism of this variation requires further study.

3. The main periods of the considered TIDs are about $72 \mathrm{~min}$ and $44 \mathrm{~min}$, respectively. For the first event the mean horizontal wave-number is $0.008 \mathrm{~km}^{-1}$ between 200 and $350 \mathrm{~km}$ altitude, corresponding to a horizontal wavelength of about $780 \mathrm{~km}$ and a phase speed of $180 \mathrm{~m} / \mathrm{s}$. At about $300 \mathrm{~km}$ altitude the vertical wavenumber is $-0.027 \mathrm{~km}^{-1}$, corresponding to a vertical wavelength of $230 \mathrm{~km}$ and a phase speed of $55 \mathrm{~m} / \mathrm{s}$. The vertical and horizontal wave vectors are directed downwards and southeastwards. These features indicate that the considered event is a moderately large-scale 
TID. The apparent horizontal wave number for the second TID is about $0.015 \mathrm{~km}^{-1}$ between 200 and $300 \mathrm{~km}$ altitude, corresponding to a horizontal wave length of about $420 \mathrm{~km}$ and a phase speed of $160 \mathrm{~m} / \mathrm{s}$. The vertical wave-number at $250 \mathrm{~km}$ altitude is about $0.005 \mathrm{~km}^{-1}$, corresponding to a vertical wavelength of $125 \mathrm{~km}$ and a phase speed of about $50 \mathrm{~m} / \mathrm{s}$. This wave propagates mainly southwestwards and can be characterized as typical medium-scale TID.

Acknowledgements. The authors thank the director and staff of EISCAT for operating the facilities and providing the data. The EISCAT Scientific Association is funded by scientific agencies of Finland (SA), France (CNRS), Germany (MPG), Japan (NIPR) Norway (NF), Sweden (NFR) and the United Kingdom (PPARC). This work is supported by China National Foundations of Natural Science. One of the authors S. Y. Ma appreciated the hospitality of the Max-Planck Institut für Aeronomie during a stay in Germany supported by the DFG.

Topical Editor D. Alcaydé thanks G. Kirchengast and B. S. Lanchester for their help in evaluating this paper.

\section{References}

Bertin, F., W. Kofman, and G. Lejeune, Observations of gravity waves in the auroral zone, Radio Sci., 18, 1059-1065, 1983.

Chen, C. H., Non-linear maximum entropy spectral analysis methods for signal recognition, Research Studies, Chichester, 1982.

Crowley, G. and I. W. McCrea, A synoptic study of TIDs observed in the United Kingdom during the first WAGS campaign, October 10-18, 1985, Radio Sci., 23, 905-917, 1988.

Folkestad, K., T. Hagfors, and S. Westerlund, EISCAT: an updated description of technical characteristics and operational capabilities, Radio Sci., 18, 867-879, 1983.

Hearn, A. L., and K. C. Yeh, Medium-scale TIDs and their associated internal gravity waves as seen through heightdependent electron density power spectra, J. Geophys. Res., 82, 4983-4990, 1977.

Hines, C. O., Internal atmospheric gravity waves at ionospheric heights, Can. J. Phys., 38, 1441-1481, 1960.

Hocke, K., Untersuchung des Phasen-und Amplitudenverhaltens von Travelling Ionospheric Disturbances mit Hilfe von EISCAT daten, PhD Thesis, University of Göttingen, 1994.

Hocke, K., and K. Schlegel, A review of atmospheric gravity waves and travelling ionospheric disturbances: 1982-1995, Annales Geophysicae, 14, 917-940, 1996.

Hocke, K., K. Schlegel, and G. Kirchengast, Phase and amplitudes of TIDs in the high-latitude F-region observed by EISCAT, J. Atmos. Terr. Phys., 58, 245-255, 1996.

Kirchengast, G., The Graz Ionospheric Flux Tube Simulation Model, in STEP Handbook of Ionospheric Models, R. W. Schunck, Ed., Logan, Utah, USA, pp 73-94, 1996a.

Kirchengast, G., Elucidation of the physics of the gravity wave-TID relationship with the aid of theoretical simulations, J. Geophys. Res., 101, 13353-13368, 1996b.

Kirchengast, G., R. Leitinger, and K. Schlegel, A high-resolution model for the ionospheric F-region at mid- and high-latitude sites, Annales Geophysicae, 10, 577-596, 1992.

Kirchengast, G., K. Hocke, and K. Schlegel, Gravity waves determined by modelling of travelling ionospheric disturbances in-incoherent-scatter radar measurements, Radio Sci., 30, 15511567,1995
Kirchengast, G., K. Hocke, and K. Schlegel, The gravity wave-TID relationship: insight via theoretical model - EISCAT data comparison, J. Atmos. Terr. Phys., 58, 233-243, 1996.

Lanchester, B. S., T. Nygen, M. J. Jarvis, and R. Edwards, Gravity wave parameters measured with EISCAT and Dynasonde, Annales Geophysicae, 11, 925-936, 1993.

Ma, S. Y. and K. Schlegel, Ionospheric responses to atmospheric gravity waves in various plasma quantities and inversion of GW's parameters. Acta Geophys. Sinica (in English), 35, (4), 467- 478, 1992.

Ma, S. Y. and K. Schlegel, Nonlinear wave-wave interactions related to gravity wave reflection in the auroral upper F-region observed by the EISCAT radar, J. Atmos. Terr. Phys., 55, 719738, 1993.

Mauelshagen, H. P., Untersuchungen an 'Traveling Ionospheric Disturbances' in der F-Schicht der Polarlichtzone mit Hilfe von EISCAT-Daten, PhD Thesis, University of Göttingen, 1990.

Morgan, M. G., Locating TID sources with a north-south chain of rapid-run ionosondes in western Quebec, Radio Sci., 18, 10661076,1983

Natorf, L., K. Schlegel, and A. W. Wernik, gravity wave parameters derived from travelling ionospheric disturbances observations in the auroral zone, Radio Sci., 27, 829-840, 1992.

Oliver, W. L., S. Fukao, T. Sato, T. Tsuda, S. Sato, I. Kimura, A. Ito, T. Saryou and T. Araki, Ionospheric incoherent scatter measurements with the middle and upper atmosphere radar: observations during the large magnetic storm of February 6-8, 1986, J. Geophys. Res., 93, 14649-14655, 1988.

Oliver, W. L., S. Fukao, M. Sato, Y. Otsuka, T. Takami and T. Tsuda, Middle and upper atmosphere radar observations of the dispersion relation for ionospheric gravity waves, J. Geophys. Res., 100, 23763-23768, 1995.

Rice, D. D., R. D. Hunsucker, L. J. Lanzerotti, G. Crowley, P. J. S. Williams, J. D. Craven, and L. Franke, An observation of atmospheric gravity wave cause and effect during the October 1985 WAGS campaign, Radio Sci., 23, 919-930, 1988.

Schlegel, K., The study of tides and gravity waves with the help of the field-aligned velocities measured by EISCAT, J. Atmos. Terr. Phys., 48, 879-886, 1986.

Sheen, D. R., and C. H. Liu, Modelling F-region gravity waves observed during the WAGS campaign-1. Special event, Radio Sci., 23, 879-893, 1988.

Shibata, T., Application of multichannel maximum entropy spectral analysis to the HF Doppler data of medium-scale TID, J. Geomagn. Geoelectr., 39, 247-260, 1987.

Strand, O. N., Multichannel complex maximum entropy (autoregressive) spectral analysis, IEEE Trans. Automat. Contr., AC22, (4) 634-640, 1977.

Ulrych, T., and O. Jensen, Cross-spectral analysis using maximum entropy, Geophys., 39, 353-354, 1974.

Williams, P. J. S., G. Crowley, K. Schlegel, T. S. Virdi, I. McCrea, G. Watkins, N. Wade, J. K. Hargreaves, T. Lanchn-Cope, H. Muller, J. E. Baldwin, P. Warner, A. P. Van Eyken, M. A. Hapgood, and A. S. Rodge, The generation and propagation of atmospheric gravity waves observed during the Worldwide Acoustic-Gravity wave Study (WAGS), J. Atmos. Terr. Phys., 50, 323-337, 1988.

Williams, P. J. S., T. S. Virdi, R. V. Lewis, M. Lester, A. S. Rodger, I. W. McCrea, and K. S. C. Freeman, Worldwide atmospheric gravity-wave study in the European sector 1985-1990, J. Atmos. Terr. Phys., 55, 683-696, 1993.

Wuttke, K., Numerical modelling of gravity waves in the ionospheric F2-region, MPAE Rep. W-05-94-26, MPI für Aeronomie, Lindau, 1994. 